\title{
The Rupture of Thin Liquid Films Placed on Solid and Liquid Substrates in Gravity Body Forces
}

\author{
A. L. Kupershtokh ${ }^{1,2, *}$, E. V. Ermanyuk ${ }^{1,3}$ and N. V. Gavrilov ${ }^{1}$ \\ ${ }^{1}$ Lavrentyev Institute of Hydrodynamics SB RAS, Novosibirsk 630090, Russia. \\ 2 National Research Novosibirsk State University, Novosibirsk 630090, Russia. \\ ${ }^{3}$ Laboratoire de physique ENS de Lyon, France.
}

Received 30 October 2013; Accepted (in revised version) 1 November 2014

\begin{abstract}
This paper presents a numerical and experimental study on hydrodynamic behavior of thin liquid films in rectangular domains. Three-dimensional computer simulations were performed using the lattice Boltzmann equation method (LBM). The liquid films laying on solid and liquid substrates are considered. The rupture of liquid films in computations is initiated via the thermocapillary (Marangoni) effect by applying an initial spatially localized temperature perturbation. The rupture scenario is found to depend on the shape of the temperature distribution and on the wettability of the solid substrate. For a wettable solid substrate, complete rupture does not occur: a residual thin liquid film remains at the substrate in the region of pseudo-rupture. For a non-wettable solid substrate, a sharp-peaked axisymmetric temperature distribution induces the rupture at the center of symmetry where the temperature is maximal. Axisymmetric temperature distribution with a flat-peaked temperature profile initiates rupture of the liquid film along a circle at some distance from the center of symmetry. The outer boundary of the rupture expands, while the inner liquid disk transforms into a toroidal figure and ultimately into an oscillating droplet.

We also apply the LBM to simulations of an evolution of one or two holes in liquid films for two-layer systems of immiscible fluids in a rectangular cell. The computed patterns are successfully compared against the results of experimental visualizations. Both the experiments and the simulations demonstrate that the initially circular holes evolved in the rectangular cell undergoing drastic changes of their shape under the effects of the surface tension and gravity. In the case of two interacting holes, the disruption of the liquid bridge separating two holes is experimentally observed and numerically simulated.
\end{abstract}

AMS subject classifications: 76M28, 76T30, 76M25, 65Z05

Key words: Lattice Boltzmann equation method, phase transition, liquid films, dynamics of multiphase media, Marangoni effect, computer simulations.

*Corresponding author. Email addresses: skn@hydro.nsc.ru (A. L. Kupershtokh), ermanyuk@gmail .com (E. V. Ermanyuk), gavrilov@hydro.nsc.ru (N. V. Gavrilov) 


\section{Introduction}

The mechanism for rupture of thin liquid films is of importance for colloid science, foam mechanics, ecological problems and many engineering applications. The films can be freely hanging as well as laying on solid or liquid substrates. The theory of the critical thickness of rupture of free thin liquid films was proposed by Scheludko et al. in [13]. There exists extensive literature on rupture of thin liquid films on solid substrates including structured surfaces (see review [4]). The systems consisting of thin liquid films on liquid substrates are widely present in nature and technology (see review [5]). One of the first experiments on the hydrodynamic behavior of thin films on liquid substrate after rupture was carried out in work [6], where the authors used a round cell and considered an axisymmetric problem of expansion and further evolution of a hole in the film.

Our earlier work [7] was devoted to three-dimensional computer LBM simulations of the dynamics of thin freely hanging liquid films with no gravity under the action of a radially symmetric temperature distribution with a maximum at the center. This temperature distribution can initiate a rupture of the film due to the thermocapillary effect. Depending on the shape of the temperature distribution in the vicinity of the symmetry axis (maximum of temperature), the film ruptured either with the formation of a central round hole or an annular hole. In the latter case, a liquid disk was formed that later evolved into a droplet in the center of the round hole.

The present study is focused on the hydrodynamic aspects of the rupture of thin liquid films on liquid and solid substrates in a rectangular cell. The evolution of one or two holes in liquid films on a liquid substrate is investigated in experiments and is simulated for two-layer systems of immiscible fluids.

The three-dimensional computer simulations are performed using the lattice Boltzmann equation method. The phase transitions in the LBM are simulated by introducing attractive forces acting on the matter at the nodes of the computation grid. The pseudopotential method proposed in $[8,9]$ describes the fluids with an arbitrary equation of state (pressure as a function of density and temperature) and simulates the interfaces between vapor and liquid phases with a surface tension. Here we use the pseudopotential model considerably improved in works [10-12]. For liquid films and liquid substrates we use the model van der Waals equation of state for which the surface tension decreases with temperature and tends to zero near the critical point. The effect of solid walls on fluids is simulated by special forces acting between the nodes representing the solid boundaries and the nodes belonging to the liquid or vapor phases.

\section{Lattice Boltzmann Equation method}

Simulations of hydrodynamic flows with the interfaces between liquids and gases are of vivid interest in scientific and industrial applications. We use the lattice Boltzmann equation method in computer simulations because it does not require tracking of the 
interfaces that appear, disappear and undergo topological changes.

Actually, the algorithm of one-component two-phase lattice Boltzmann method includes the following steps:

- Choice of regular spatial lattice and corresponding pseudo-particle velocity set;

- Transfer of distribution functions (pseudo-particles) along the characteristics;

- Implementation of a collision operator;

- Implementation of the body forces;

- Use of specific equations of state for fluids;

- Fulfillment of the criterion for stability;

- Implementation of boundary conditions.

This method describes the fluid flows as a motion of pseudo-particles having a certain distribution over a discrete set of velocities $\mathbf{c}_{k}$. The lattice Boltzmann method describes fluids with an arbitrary prescribed equation of state and simulates the vapor-liquid interfaces with a surface tension. In LBM, the different phases of a substance (liquid and vapor) are usually simulated as one fluid. For this purpose, special forces acting on a fluid in nodes of the computation grid are introduced. The global momentum conservation law requires that these forces should be a gradient of a certain pseudopotential. This idea of the pseudopotential was firstly proposed in work [8] for equations of state given in the form $P(\rho)$ and then extended in [9] to the case with $P(\rho, T)$, where $P, \rho$ and $T$ are the pressure, density and temperature, respectively. Later, this model was considerably improved in works [10-12].

In the lattice Boltzmann method, the single-particle distribution functions $N_{k}(\mathbf{x}, t)$ are used as variables in accordance with the chosen finite set of velocity vectors $\mathbf{c}_{k}, k=0, \cdots, b$. The evolution equation for the distribution functions $N_{k}$ can be written in the form

$$
N_{k}\left(\mathbf{x}+\mathbf{c}_{k} \Delta t, t+\Delta t\right)=N_{k}(\mathbf{x}, t)+\Omega_{k}(N)+\Delta N_{k},
$$

where $\Delta t$ is the time step, $\Omega_{k}$ is the collision operator, and $\Delta N_{k}$ is the change of the distribution functions due to the action of the internal and external body forces.

The hydrodynamic variables (the density $\rho$ and the velocity $\mathbf{u}$ of fluid) in a node are calculated as

$$
\begin{aligned}
& \rho=\sum_{k=0}^{b} N_{k}, \\
& \rho \mathbf{u}=\sum_{k=1}^{b} \mathbf{c}_{k} N_{k} .
\end{aligned}
$$

The vectors $\mathbf{c}_{k}$ of pseudo-particle velocity depend on the chosen lattice. For threedimensional simulations, the three-dimensional version of lattice Boltzmann method 
D3Q19 with nineteen vectors $\mathbf{c}_{k}$ of pseudo-particle velocity on cubic lattice [13] is realized. Hence, we have for velocity vectors $\left|\mathbf{c}_{0}\right|=0,\left|\mathbf{c}_{1-6}\right|=h / \Delta t$, and $\left|\mathbf{c}_{7-18}\right|=\sqrt{2} h / \Delta t$. Here, $h$ is the lattice spacing.

The collision operator is usually used in Bhatnagar-Gross-Krook (BGK) form [14]

$$
\Omega_{k}=\left(N_{k}^{e q}(\rho, \mathbf{u})-N_{k}(\mathbf{x}, t)\right) / \tau,
$$

where $\tau$ is the dimensionless relaxation time. Nevertheless, the multi-relaxation time (MRT) collision operator also can be used. For BGK model, the kinematic viscosity depends on the relaxation time in accordance with the well-known expression $v=$ $\theta(\tau-1 / 2) \Delta t$. Here $\theta$ is the "kinetic temperature" of LBM pseudo-particles. For the lattice Boltzmann models D1Q3, D2Q9 and D3Q19 the value $\theta=(h / \Delta t)^{2} / 3$ is usually used.

The Exact Difference Method (EDM) [15-17] is used for the implementation of the body forces (internal forces and gravity) in the LBM:

$$
\Delta N_{k}(\mathbf{x}, t)=N_{k}^{e q}(\rho, \mathbf{u}+\Delta \mathbf{u})-N_{k}^{e q}(\rho, \mathbf{u}),
$$

where the value of the velocity after the action of the total force $\mathbf{F}$ on a node is equal to $\mathbf{u}+\Delta \mathbf{u}=\mathbf{u}+\mathbf{F} \Delta t / \rho$.

The corresponding equilibrium distribution functions [18] are calculated as

$$
N_{k}^{e q}(\rho, \mathbf{u})=\rho w_{k}\left(1+\frac{\mathbf{c}_{k} \mathbf{u}}{\theta}+\frac{\left(\mathbf{c}_{k} \mathbf{u}\right)^{2}}{2 \theta^{2}}-\frac{\mathbf{u}^{2}}{2 \theta}\right) .
$$

For fluids, the van der Waals equation of state is used. In terms of reduced variables $\tilde{P}=P / P_{\text {cr }}, \tilde{\rho}=\rho / \rho_{\text {cr }}$ and $\tilde{T}=T / T_{\text {cr }}$, the equation has the form

$$
\tilde{P}=\frac{8 \tilde{\rho} \tilde{T}}{3-\tilde{\rho}}-3 \tilde{\rho}^{2} .
$$

Here $P_{\mathrm{cr}}, \rho_{\mathrm{cr}}$, and $T_{\mathrm{cr}}$ are the values of pressure, density and temperature at the critical point.

For equations of state in the form $P(\rho)$, the total force $\mathbf{F}$ acting on a node can be calculated as a gradient of pseudopotential $\mathbf{F}=-\nabla U$ [8]. If the equation of state has the form $P(\rho, T)$, the pseudopotential $U$ can be used in the slightly modified form $U(\rho, T)=$ $P(\rho, T)-\rho \theta[9]$.

In [10-12], a new special function $\Phi(\rho, T)=\sqrt{-U}$ has been proposed. Hence, the total force can be written in the new equivalent form

$$
\mathbf{F}=2 A \nabla\left(\Phi^{2}\right)+2(1-2 A) \Phi \nabla \Phi .
$$

Here $A$ is the free parameter $[10,11]$. The appropriate isotropic finite difference approximation of this equation was proposed in [10-12]:

$$
\mathbf{F}(\mathbf{x})=\frac{1}{\alpha h}\left[A \sum_{k=1}^{b} G_{k} \Phi^{2}\left(\mathbf{x}+\mathbf{e}_{k}\right) \mathbf{e}_{k}+(1-2 A) \Phi(\mathbf{x}) \sum_{k=1}^{b} G_{k} \Phi\left(\mathbf{x}+\mathbf{e}_{k}\right) \mathbf{e}_{k}\right] .
$$


For the three-dimensional model D3Q19, the coefficient $\alpha$ is equal to 3, and the coefficients $G_{k}>0$, with $G_{1-6}=1$ and $G_{7-18}=1 / 2$ for basic and diagonal directions of the cubic lattice, respectively.

For the van der Waals equation of state, the free parameter $A$ is approximately equal to $-0.152[10,11]$ to ensure the relevant values of vapor density on the coexistence (binodal) curve.

To simulate the immiscible fluids, two sets of the LBM distribution functions $N_{k}^{s}$ and $N_{k}^{\sigma}$ are used. The evolution equations for distribution functions of each component $s$ and $\sigma$ have the form

$$
N_{k}^{s, \sigma}\left(\mathbf{x}+\mathbf{c}_{k} \Delta t, t+\Delta t\right)=N_{k}^{s, \sigma}(\mathbf{x}, t)+\Omega_{k}^{s, \sigma}+\Delta N_{k}^{s, \sigma} .
$$

To ensure immiscibility of the two fluids, the special forces of interaction between components are introduced. These repulsive forces act on the substance of each component in a node $i$ from the substance of the other component that is present in neighbor nodes $k$. The total forces acting on the substance of components at a node due to these repulsion forces have the form

$$
\begin{aligned}
& \mathbf{F}^{\mathcal{S}(\mathbf{x})}=\psi\left[\rho^{s}(\mathbf{x})\right] \sum_{k=1}^{b} B_{k} \psi\left[\rho^{\sigma}\left(\mathbf{x}+\mathbf{e}_{k}\right)\right] \mathbf{e}_{k}, \\
& \mathbf{F}^{\sigma}(\mathbf{x})=\psi\left[\rho^{\sigma}(\mathbf{x})\right] \sum_{k=1}^{b} B_{k} \psi\left[\rho^{S}\left(\mathbf{x}+\mathbf{e}_{k}\right)\right] \mathbf{e}_{k} .
\end{aligned}
$$

Here, $\psi(\rho)$ is the increasing positive function that depends on the density of the corresponding component. Obviously, the value $\psi(0)$ should be equal to zero. In the present work, we use the model with a linear law of interaction $\psi(\rho)=\rho$ as the reasonable approximation that is widely used in LBM simulations to ensure an immiscibility of liquids [19]. All coefficients $B_{k}$ are proportional to one parameter $B<0$, that describes the intensity of interaction between components.

At the walls and at the bottom of the cell the impermeability boundary conditions are prescribed. We use the quite simple well-known "bounce-back" rule to implement in the LBM no-slip boundary conditions at the solid bottom of the cell. In the case of a nonwettable substrate, it practically does not slow down the flow. At the solid walls of the cell, we use the well-known entirely slip-flow boundary conditions to reduce the influence of boundary layers. Note that the "bounce back" boundary conditions in LBM do not ensure the fully no-slip boundary conditions if the BGK collision operator is used.

The degree of wettability of the walls and of the bottom of the cell by both fluids $s$ and $\sigma$ are simulated by the special forces $\mathbf{F}_{\mathrm{bc}}$ acting between the nodes representing the solid boundaries and the nodes belonging to the liquid or vapor phases

$$
\mathbf{F}_{\mathrm{bc}}^{s}(\mathbf{x})=\psi\left[\rho^{s}(\mathbf{x})\right] \sum_{k=1}^{b} C_{k} \psi\left[\rho_{\text {solid }}\left(\mathbf{x}+\mathbf{e}_{k}\right)\right] \mathbf{e}_{k}
$$




$$
\mathbf{F}_{\mathrm{bc}}^{\sigma}(\mathbf{x})=\psi\left[\rho^{\sigma}(\mathbf{x})\right] \sum_{k=1}^{b} C_{k} \psi\left[\rho_{\text {solid }}\left(\mathbf{x}+\mathbf{e}_{k}\right)\right] \mathbf{e}_{k} .
$$

Here, $\rho_{\text {solid }}$ is the parameter that allows one to control the value of wettability of the solid surfaces ( $\rho_{\text {solid }} \neq 0$ only for nodes belonging to the solid boundaries). On the contrary, the densities of fluid components $s$ and $\sigma$ are equal to zero for all nodes belonging to the solid boundaries. All coefficients $C_{k}$ are proportional to one parameter of interaction between the liquid and solid surfaces $C<0$. In this case, the product $C \psi\left(\rho_{\text {solid }}\right)$ is only important because it describes the wettability of the solid walls [19].

In several simulations (Section 3), we also used the periodic boundary conditions along the $x$ and $y$ directions.

For LBM with an equation of state known in the form $P(\rho, T)$, the criterion for a numerical stability has been derived in [17]

$$
\left(\frac{\partial \tilde{P}}{\partial \tilde{\rho}}\right)_{T} \leq(1+\tilde{\theta})\left(\frac{h}{\Delta t}\right)^{2} .
$$

Here, $\tilde{\theta}=c_{s}^{2}=1 / 3$ is the dimensionless "kinetic temperature" of LBM pseudoparticles and $c_{s}$ is the lattice sound speed in classical LBM. Hence, the time step $\Delta t$ and the lattice spacing $h$ should agree with the criterion (2.13). The speed of sound $(\partial P / \partial \rho)_{T}$ in liquid phase should be calculated from the equation of state.

In spite of the constant value of the dimensionless "kinetic temperature" of LBM pseudoparticles $\tilde{\theta}=1 / 3$ in "isothermal" model of LBM, the temperature $\tilde{T}$ in the equation of state determines the value of the surface tension. In the present paper, we only used the property of a surface tension to change its value in accordance with a prescribed temperature distribution $T(\mathbf{x}, t)$.

The reduced values of the surface tension $\tilde{\sigma}=\sigma /\left(P_{\mathrm{cr}} h\right)$ (Fig. 1) were calculated in accordance with Laplace's law for two-dimensional case $\tilde{P}_{L}-\tilde{P}_{V}=\tilde{\sigma} / \tilde{R}$ [20] for round liquid droplets in a saturated vapor. Here $\tilde{P}_{L}$ is the reduced pressure in the liquid droplet, $\tilde{P}_{V}$ is the reduced pressure in saturated vapor, and $\tilde{R}$ is the reduced droplet radius. For the given equation of state, the values of $\tilde{\sigma}$ obtained in two-dimensional and three-dimensional simulations are the same. The surface tension in the lattice Boltzmann method depends also on the dimensionless parameter

$$
k=\frac{P_{\mathrm{cr}}}{\rho_{\mathrm{cr}}}\left(\frac{\Delta t}{h}\right)^{2} .
$$

We used and tested our version of LBM for many years [7,10-12,15-17,20]. We have shown that our model describes the coexistence curve (binodal) for several equations of state extremely well [10] (much better than many other variants of LBM), including the real (International) equation of state for water [20]. Also, it has been shown that our model describes the liquid droplet shape oscillations in good agreement with theoretical estimations [20]. 


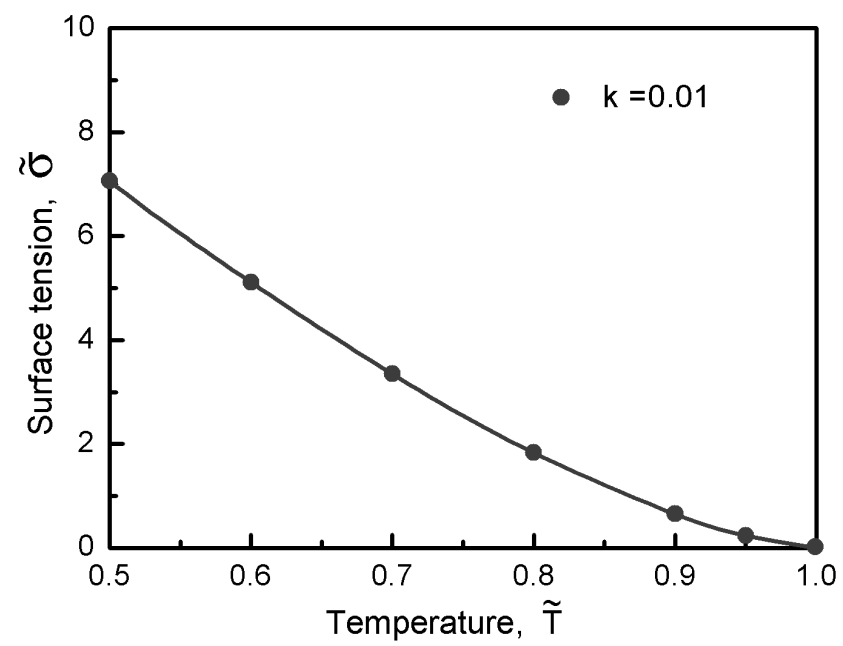

Figure 1: Reduced surface tension vs. temperature for the van der Waals equation of state. The dimensionless parameter $k=0.01$.

For all computer simulations the Graphics Processing Units (GPUs) are exploited. We use the GPU with "Fermi" architecture GTX-580 (512 cores, 3 GB device memory) and also with "Kepler" architecture GTX-Titan-Black (2880 cores, 6 GB device memory). For parallel programming we use the CUDA (Compute Unified Device Architecture) technology.

\section{Thin liquid films on a solid substrate}

It is well known that hydrodynamic flows can arise in liquids due to the dependence of surface tension on temperature. This phenomenon is known as the thermocapillary (Marangoni) effect. The three-dimensional computer simulations of thermocapillary rupture in the thin liquid films on both nonwettable and wettable solid substrates are carried out. The reason for these temperature distributions can be of different nature and is not considered in the paper. Periodic boundary conditions are used along the $x$ and $y$ directions.

\subsection{Rupture of thin liquid films at the center of a hot spot}

For the prescribed axisymmetric temperature distribution along the film surface of the form (Fig. 2a)

$$
T= \begin{cases}T_{0}+\Delta T(1+\cos (\pi r / R)), & \text { for } r<R, \\ T_{0} & \text { for } r \geq R,\end{cases}
$$

a diverging flow in the film in the vicinity of the hot spot is generated because of the gradients of the surface tension along the film. Here $r=\sqrt{x^{2}+y^{2}}$ and $R$ is the radius of spot 

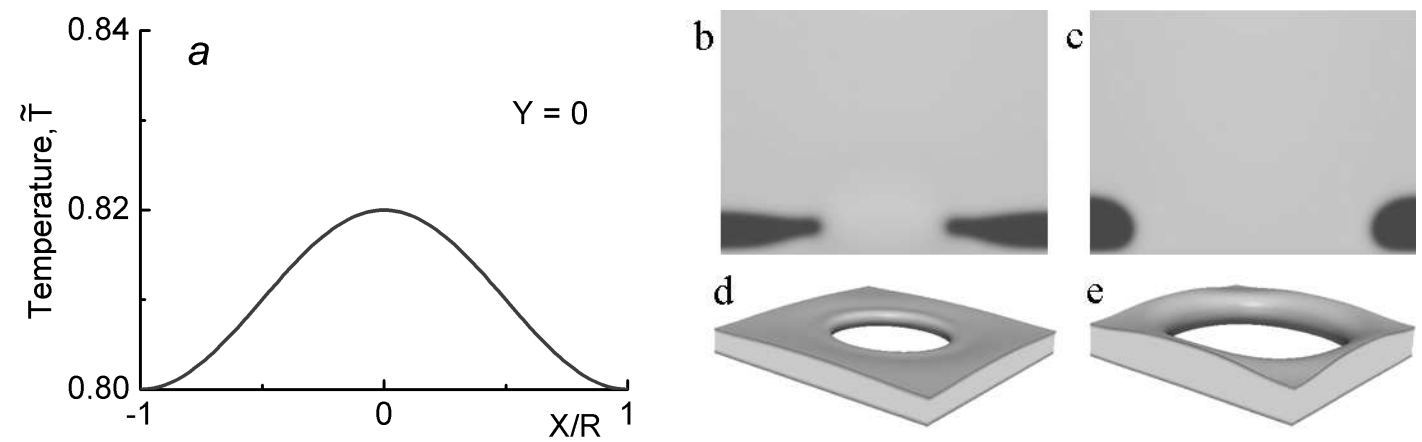

Figure 2: The temperature distribution (a) along the $x$ axis at $y=0$. The rupture of the liquid film on the nonwettable flat surface. (b) and (c) are the density distributions in the central vertical section. $\tilde{T}=0.8$, $\Delta \tilde{T}=0.01 . t=2000(\mathrm{~b}, \mathrm{~d}) ; 3200(\mathrm{c}, \mathrm{e})$. Lattice is $256 \times 256 \times 192 . R=128 h$.
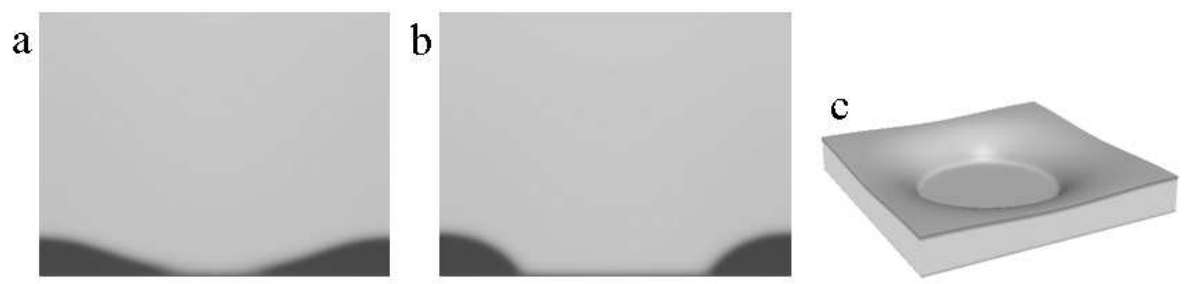

Figure 3: The stretching (pseudo-rupture) of the thin liquid film on the wettable substrate. (a) and (b) are the density distributions in the central vertical section. $t=3000(a) ; 5000(b, c)$. Lattice is $256 \times 256 \times 192$.

where the temperature is enhanced. Later, the rupture of the film occurs in the center of symmetry where the temperature is maximal (Fig. 2). Hereinafter, in the presentation of the computational results, we use the lattice time step $\Delta t$ as the time unit. For nonwettable surface, the rupture of the liquid film is complete with the formation of a hole (dry spot).

In the case of the wettable surface, the complete breakdown of the liquid film is not observed because the solid surface remains wetted by the extremely thin layer of liquid (wetted spot, precursor) (Fig. 3).

\subsection{Rupture of thin liquid films under the action of temperature distribution with a flattened vertex}

For the prescribed axisymmetric temperature distribution with a flattened vertex (Fig. 4a):

$$
T= \begin{cases}T_{0}+\Delta T\left(1+\cos \left(\pi(r / R)^{2}\right)\right), & \text { for } r<R \\ T_{0}, & \text { for } r \geq R\end{cases}
$$



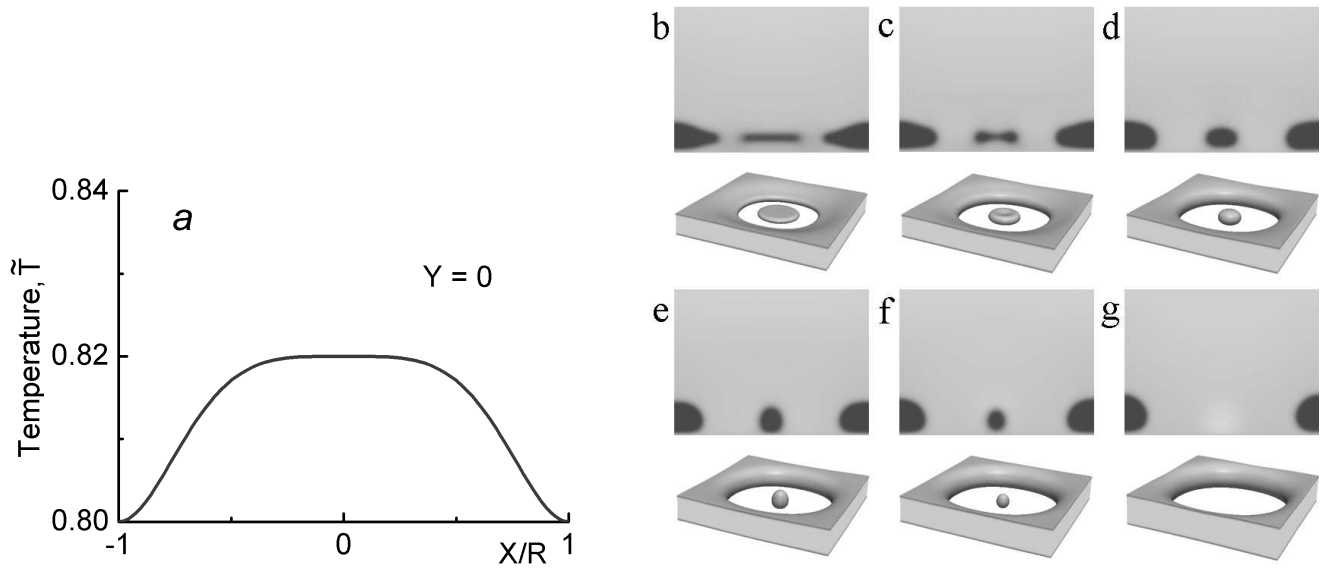

Figure 4: The temperature distribution (a) along the $x$ axis at $y=0$. The rupture of the liquid film on the nonwettable substrate. $\tilde{T}=0.8, \Delta \tilde{T}=0.01 . t=2400$ (b); 2600 (c); 2800 (d); 3000 (e); 3200 (f); 3600 (g). Lattice is $256 \times 256 \times 192$. $R=128 h$.
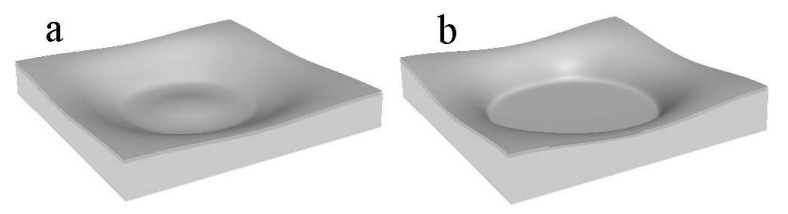

Figure 5: The stretching (pseudo-rupture) of the thin liquid film on the wettable substrate. $t=3200$ (a); 4000 (b). Lattice is $256 \times 256 \times 192$.

the rupture of the liquid film on the nonwettable substrate occurs not in the center of symmetry but along some circle where the gradient of the temperature is more pronounced. As a result, a central liquid disk is formed (Fig. 4b). The disk transforms initially into the toroidal figure due to surface tension (Fig. 4c) and then into an oscillating droplet (Fig. $4 \mathrm{~d}, \mathrm{e}$ ). Later, the gradual evaporation of the droplet takes place (Fig. 4f,g). These similar processes have been observed earlier for freely hanging films [7].

For a wettable surface of the solid substrate, full rupture does not occur. The residual thin liquid film (precursor) covers the region of pseudo-rupture. On the wettable substrate, a certain thinning of the film is formed initially in an annular region located at a certain distance from the symmetry axis, but the central "droplet" is not formed completely (Fig. 5). Note that the oscillations similar to droplet oscillations on nonwettable substrates are impossible in the case shown in Fig. 5 because of the surface tension along the wettable substrate and high shear.

\section{Thin liquid films on a layer of immiscible liquid}

The systems consisting of thin liquid films on liquid substrates are widely present in nature and technology. One of the first experiments on the rupture of thin films on liquid 
substrates was carried out in [6], where the authors used a round cell and considered only the axisymmetric expansion and further evolution of the hole. Our experiments and computer simulations are devoted to the hydrodynamic aspects of a rupture of thin liquid films on liquid substrates in a rectangular cell. In this case, the hydrodynamic flows are very complicated, not trivial and have not been well studied yet.

\subsection{Experiments}

The experiments with a two-layer system of immiscible liquids were carried out both for the evolution of a single hole and for the interaction of two holes (Fig. 6). The dynamics of the rupture of thin liquid films were studied in a horizontal rectangular cell with wettable walls and bottom. A lower layer of heavy liquid (perfluoro-n-octane $\mathrm{C}_{8} \mathrm{~F}_{18}$ ) with a density of $1.76 \mathrm{~g} / \mathrm{cm}^{3}$ was located at the bottom of the cell (thickness $20 \mathrm{~mm}$ ). The layer of heavy liquid played the role of a liquid substrate. A thin film of colored water (density of $1 \mathrm{~g} / \mathrm{cm}^{3}$ ) was slowly poured onto this liquid substrate between the left wall and the

a) Side view

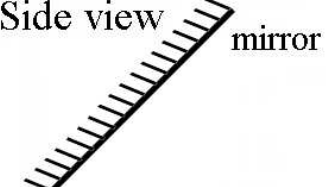

After pouring water layer onto substrate
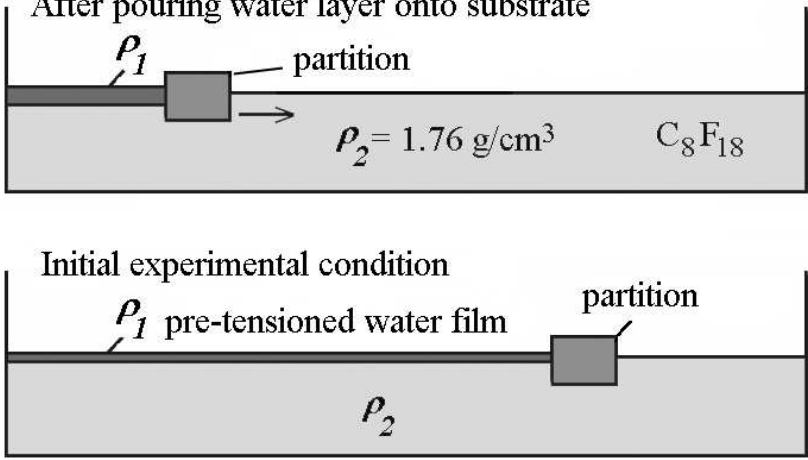

b) View from the top
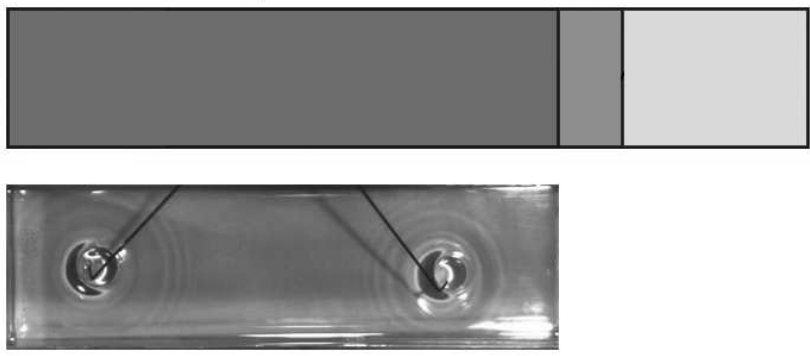

Figure 6: Experimental setup.

\section{Video camera}

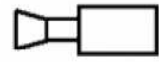



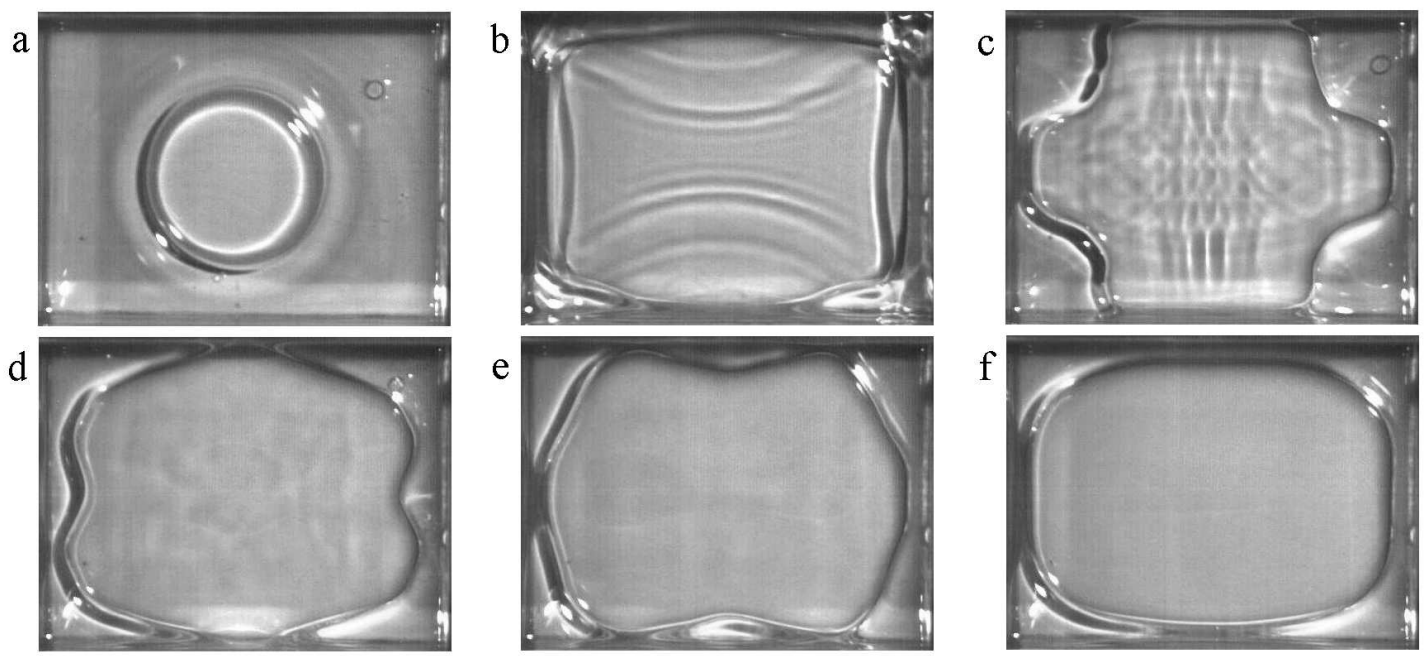

Figure 7: The rupture of the thin liquid film on the surface of heavier liquid substrate. Aspect ratio $A R=1.29$. $t=0.24 \mathrm{~s}(\mathrm{a}), 0.55 \mathrm{~s}(\mathrm{~b}), 0.80 \mathrm{~s}(\mathrm{c}), 1.46 \mathrm{~s}(\mathrm{~d}), 1.94 \mathrm{~s}(\mathrm{e}), 3.62 \mathrm{~s}(\mathrm{f})$. The dimensionless time $t_{\exp }=0.76(\mathrm{a})$, 1.74 (b), 2.52 (c), 4.6 (d), 6.1 (e), 11.4 (f).

partition. Then the partition could be moved toward the right to produce the thin film of water. Thus, the aspect ratio of liquid film and its thickness could be varied.

The rupture of the pre-tensioned water film in the central region was initiated by one or two non-wettable thin needles. In the case of two needles, the onsets of rupture at each needle were simultaneous with good accuracy. The evolution of the liquid film with holes was video-recorded using the high speed camera MotionXtra-HG-100K. The frame rate was varied from 100 to 150 frames per second.

The holes are observed to expand due to the action of surface tension and interaction with the wettable walls. The shape of the holes changes drastically (Figs. 7 and 8). The time elapsed after the onset of rupture is indicated in captions to these figures. The dimensionless time $t_{\exp }=t / t_{0}$ is introduced for experimental data using the time scale $t_{0}=\sqrt{\rho L^{2} d / \sigma}$ as was done in [21].

The main parameter for the problem is the Bond number $\mathrm{Bo}=\rho g d^{2} / \sigma$. It represents a dimensionless parameter used to characterize the ratio of gravity force to surface tension force. The Bond number is equal to 0.3 for the experiment shown in Fig. 7. Here $\rho$ is the density of water film, $d=1.5 \mathrm{~mm}$ is the initial thickness of the film, $\sigma$ is the surface tension of water, and $g$ is the acceleration of gravity.

The second parameter $\sqrt{\mathrm{La}}=\sqrt{L \rho \sigma} / \mu$ is the Reynolds number $\operatorname{Re}=\rho V L / \mu$ divided by the square root of the Weber number $\sqrt{\mathrm{We}}=\sqrt{\rho V^{2} L / \sigma}$. The parameter $\sqrt{L \rho \sigma} / \mu$ compares the capillary and viscous forces and is usually used for liquid droplets and films. Here $L=70 \mathrm{~mm}$ is the width of the cell and $\mu$ is the dynamic viscosity of water. The parameter $\sqrt{\mathrm{La}}$ was of order of $2 \cdot 10^{3}$ in the experiments. 

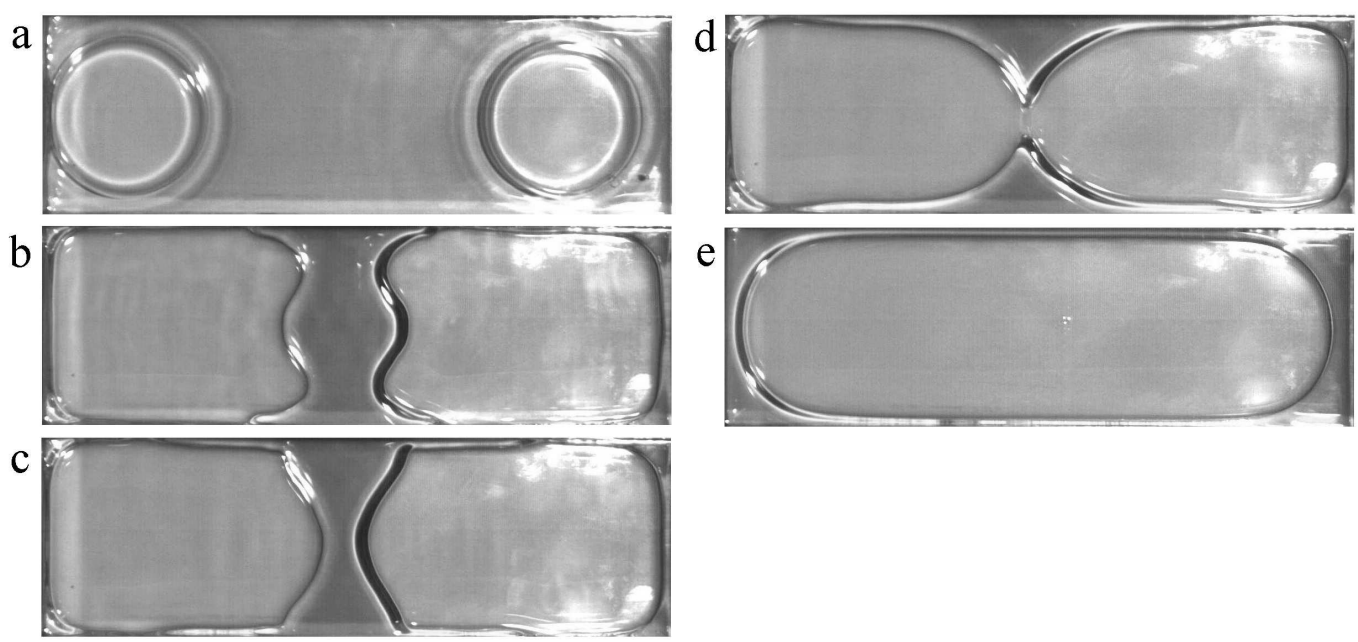

Figure 8: Merging of two holes in a thin liquid film on the surface of heavier liquid substrate. $t=0.32 \mathrm{~s}(\mathrm{a})$, $1.72 \mathrm{~s}(\mathrm{~b}), 2.32 \mathrm{~s}(\mathrm{c}), 3.38 \mathrm{~s}(\mathrm{~d}), 11.43 \mathrm{~s}(\mathrm{e})$. The dimensionless time $t_{\exp }=1.21$ (a), 6.49 (b), 8.75 (c), 12.8 (d), $43.1(\mathrm{e})$.

The expansion and further evolution of the hole in the film are not axisymmetric in contrast with the experiments that were carried out in a round cell [6].

In the case of the interaction of two holes, the disruption of the liquid bridge separating the two holes is observed. At the final stage, the shape of the resulting hole tends to the stationary oval form (Fig. 8). The initial thickness of the film for this experiment is $d=1 \mathrm{~mm}$ and the width of the cell is $L=70 \mathrm{~mm}$. Hence, the Bond number is equal to 0.13 . The time scale is $t_{0}=0.265 \mathrm{~s}$.

\subsection{Numerical simulations of liquid films on a solid nonwettable substrate}

Three-dimensional computer simulations of the rupture of liquid films on the surface of liquid substrate in a rectangular cell were carried out. The gravity plays an important role in this process. As a first reasonable approximation of the two-layer problem, the single film on a nonwettable bottom with wettable walls under gravity can be considered as a model system. A more complicated problem is the behavior of a system of two liquid layers.

At the first stage of simulations, the surface of a flat film of constant thickness evolves to the self-consistent form with menisci at the walls and corners (9000 time steps). For the sake of simplicity, the rupture of the liquid film is initiated by a prescribed short temperature pulse applied to a small localized area. In the vicinity of the hot spot, diverging flow is generated due to the Marangoni effect which triggers the rupture of the film. The rupture being initiated, the temperature is immediately set to the initial uniform value to avoid any effect of the temperature on the subsequent evolution of the flow. 

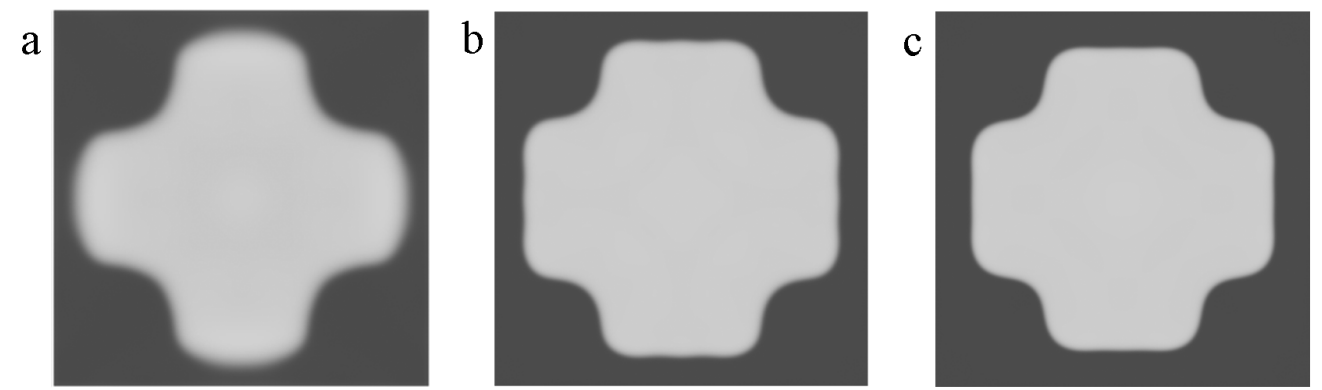

Figure 9: Convergence test for the rupture of a thin liquid film in the square cell with wettable walls and nonwettable bottom. $t=3600$ [9000] (a), 6400 [8400] (b), 10400 (c) time steps. Lattice is $192 \times 192 \times 112$ (a), $368 \times 368 \times 112$ (b), $480 \times 480 \times 112$ (c).

The results of three-dimensional simulations for a single hole in the film are shown in Figs. 9 and 10. Perfect symmetry can be obtained in square cell (Fig. 9), if the center of the hole is placed exactly in the center of the cell.

The Bond number in reduced variables $\mathrm{Bo}=\tilde{\rho} \tilde{g} \tilde{d}^{2} / k \tilde{\sigma}$ is kept constant in the simulations shown in Fig. 9. The Bond number is approximately equal to 0.8. Here $\tilde{d}=16$ is the initial thickness of the film. The reduced density of liquid film $\tilde{\rho}$ is equal to 1.93 . The dimensionless value of surface tension is equal to $\tilde{\sigma}=1.8$ for $\tilde{T}=0.8$ (Fig. 1 ). The reduced acceleration of gravity $\tilde{g}=g \Delta t^{2} / h$ is chosen equal to 0.00003 . The dimensionless parameter $k=0.01$ is introduced specifically to apply the reduced variables in LBM [20] (Section 3).

The $\sqrt{\mathrm{La}}$ number in reduced variables has the form

$$
\sqrt{\mathrm{La}}=\frac{3}{\tau-0.5} \sqrt{\frac{\tilde{L} k \tilde{\sigma}}{\tilde{\rho}}}
$$

and is kept constant in these simulations by varying the relaxation time $\tau(0.528,0.54$ and $0.546)$ for different values of the dimensionless width of the cell $\tilde{L}=L / h(192,368$ and 480).

Reasonable convergence is demonstrated for the simulations with coarse (Fig. 9a), intermediate (Fig. 9b) and fine (Fig. 9c) grid spacing $(192 \times 192 \times 112,368 \times 368 \times 112$ and $480 \times 480 \times 112$, respectively). For the fine grid, the advance in time is slow since the time step in LBM $\Delta t$ is proportional to the grid spacing $h$. Hence, the 3600 time units for the coarse grid (Fig. 9a) and 6400 time units for the intermediate grid (Fig. 9b) correspond to the 9000 and 8400 time units for the fine grid, respectively. Because of the finite size of the meniscus (of order 10 nodes) compared with the size of the domain, the results obtained with the coarse grid are not good. However the results with the intermediate and fine grids show very similar patterns at similar moments of time.

The simulations are carried out also for a rectangular cell with aspect ratio $A R=1.26$ (Fig. 10). The initial thickness of the film is equal to $\tilde{d}=16$. The reduced density of the 

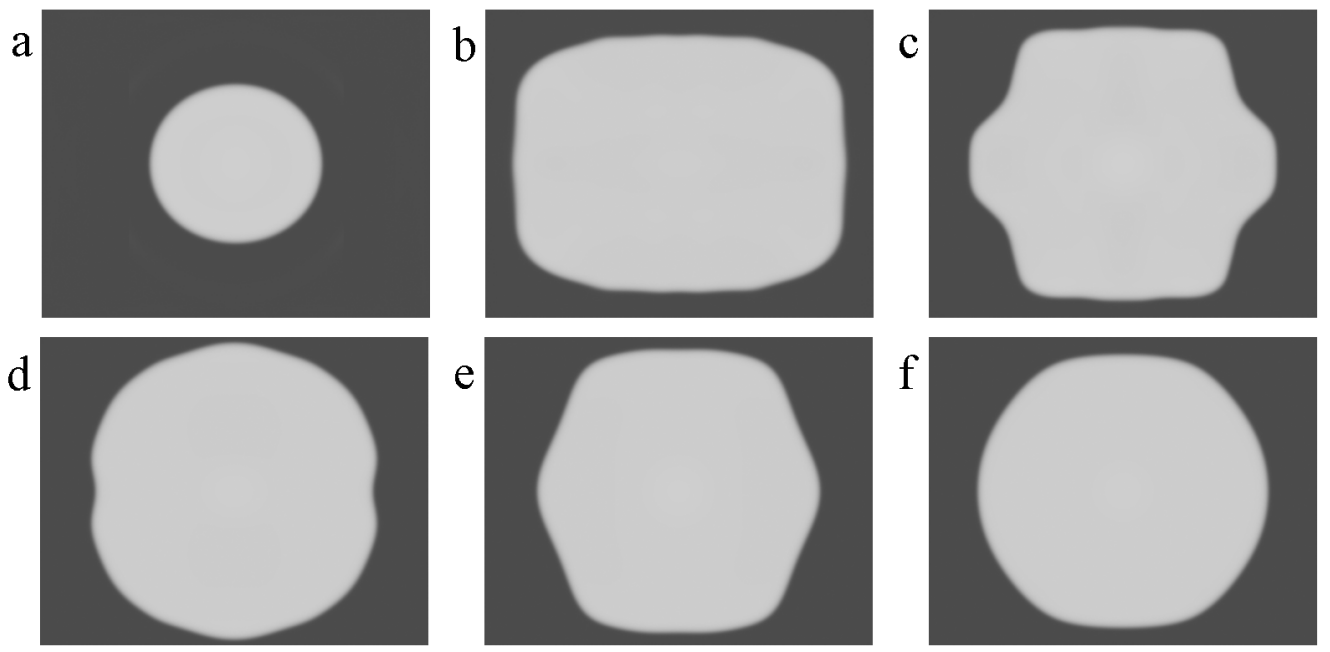

Figure 10: Patterns of the rupture of the thin liquid film in rectangular cell with wettable walls and nonwettable bottom. Aspect ratio $A R=1.26 . \quad t_{\mathrm{sim}}=0.09(\mathrm{a}), 0.41$ (b), $0.6(\mathrm{c}), 1.1$ (d), 1.3 (e), 2.7 (f). Lattice is $464 \times 368 \times 128$.

liquid film $\tilde{\rho}$ is equal to 1.93 . The dimensionless value of surface tension is equal to $\tilde{\sigma}=1.8$. The reduced acceleration of gravity $\tilde{g}$ is chosen equal to 0.000015 . The dimensionless parameter $k=0.01$. The Bond number is approximately equal to 0.4 which is close to the one for the experiment shown in Fig. 7.

A qualitative agreement of the simulations (Fig. 10) with the results of the hydrodynamic experiments (Fig. 7) is observed. The time scale in reduced variables has the form $\tilde{t}_{0}=\sqrt{\tilde{\rho} \tilde{L}^{2} \tilde{d} /(k \tilde{\sigma})}$. Hence, we have dimensionless time $t_{\text {sim }}=t / \tilde{t}_{0}$ for simulations. The ratio of dimensionless time of frames in simulations $t_{\text {sim }}$ (Fig. 10) to dimensionless time of experimental frames $t_{\exp }$ (Fig. 7) is shown in Fig. 11. The coefficient (slope of the straight line) is approximately equal to 0.23 .

The $\sqrt{\text { La }}$ number was on the order of 100 in the simulations which is considerably less than the experimental one. Unfortunately, we can not considerably enlarge this parameter in LBM simulations to ensure high values of this parameter to correspond to experimental values. For this purpose, one should either reduce the value of viscosity (by varying the relaxation time) or enlarge the domain grid. The first way is limited by the numerical stability of LBM, and the second way is limited by the amount of global memory in GPUs. As a result, the values of $t_{\text {sim }}$ for selected frames of typical shapes shown in Fig. 10 have the some uncertainties in time of about $20-30 \%$ on Fig. 11. However, keeping in mind the mentioned difference in time-scales, the evolution of the film in simulations is similar to the experimentally observed one because the main parameter for such kind of film flows is the Bond number.

In the case of two holes in the film (Fig. 8), the film behavior (the breakdown of the 


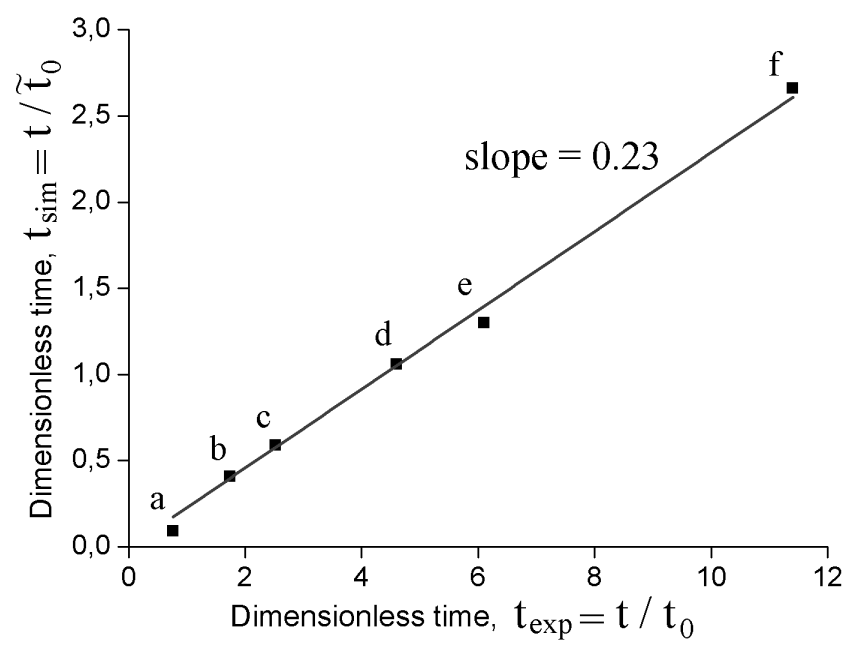

Figure 11: Dimensionless time of frames in simulations $t_{\text {sim }}$ vs. dimensionless time of experimental frames texp.
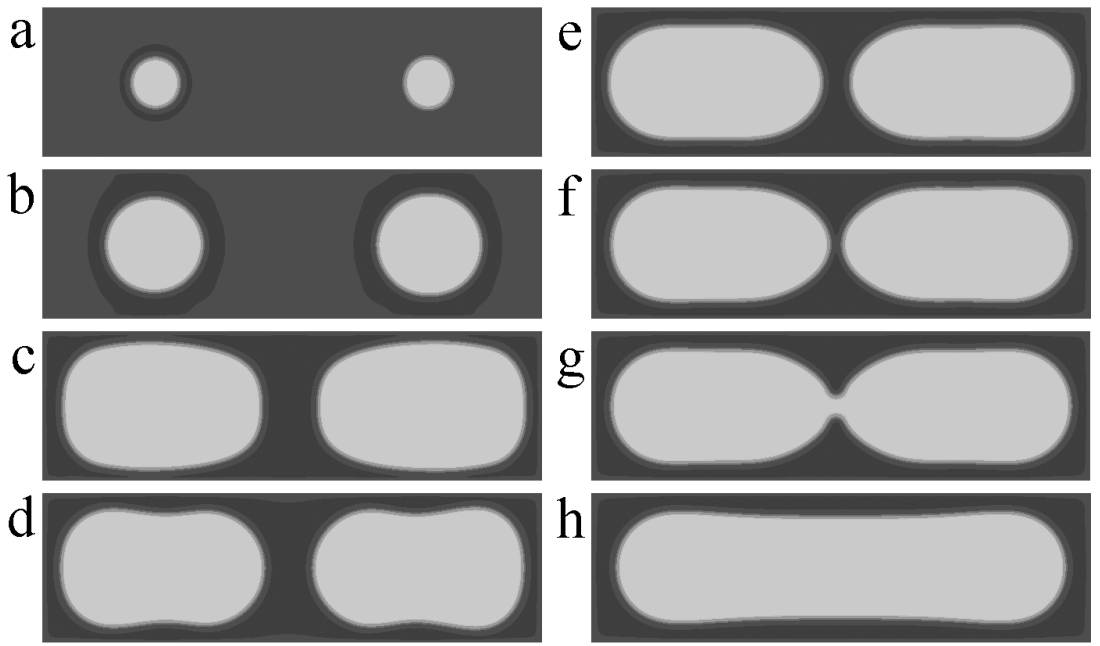

Figure 12: Merging of two holes in the film. $t_{\text {sim }}=0.28$ (a), 0.39 (b), 0.75 (c), 1.4 (d), 2.2 (e), 2.9 (f), 3.0 (g), $7.2(\mathrm{~h})$. Lattice is $640 \times 192 \times 80$.

liquid bridge separating holes) is reproduced qualitatively (Fig. 12). The initial thickness of the film is equal to $\tilde{d}=12$. The reduced acceleration of gravity $\tilde{g}$ is chosen equal to 0.000033. Hence, the time scale for this simulation is $\tilde{t}_{0}=6900$. The Bond number is approximately equal to 0.5 .

The ratio of moments of time corresponding to the breakdown of the liquid bridges separating the holes in simulation and in experiment (Fig. 8d and Fig. 12g) is about $1 / 4$ which is close to the value obtained in Fig. 11. 
The main difference with the experiment shown in Fig. 8 arises near the side walls because of the meniscus effect. The limited grid resolution did not allow us to describe it better in simulations. However, even the qualitative results obtained in computer simulations are very useful and allow one to understand many physical features of the system simulated.

\subsection{Numerical simulations of liquid films on a liquid substrate}

The results of computer simulations of the rupture of the films on the liquid substrate are shown in Figs. 13 and 14. The LBM with two components has been used for simulation of the more complicated two-layer system of immiscible liquids. For each component, the different set of LBM distribution functions is used. For the two-layer system, the cell bottom is considered also as wettable.

The reduced density of liquid film $\tilde{\rho}$ is equal to 1.93 . The dimensionless value of surface tension is equal to $\tilde{\sigma}=1.8$. The reduced acceleration of gravity $\tilde{g}$ is chosen equal to 0.000033 . The dimensionless parameter $k=0.01$. The initial thicknesses of the films $\tilde{d}$ are equal to 14 and 25 for simulations shown in Figs. 13 and 14, respectively. The corresponding Bond numbers are approximately equal to 0.7 and 2 . The parameter $\sqrt{\mathrm{La}}$ is approximately equal to 7 in these simulations at the values of the relaxation time $\tau=1.0$ and of the cell width $\tilde{L}=160$.

Owing to the effect of viscosity, the fluid motion in the thin film generates motion in the liquid substrate. The interface of the liquid substrate does not remain flat, but the accommodation occurs because of gravity and surface tension (see Fig. 13).
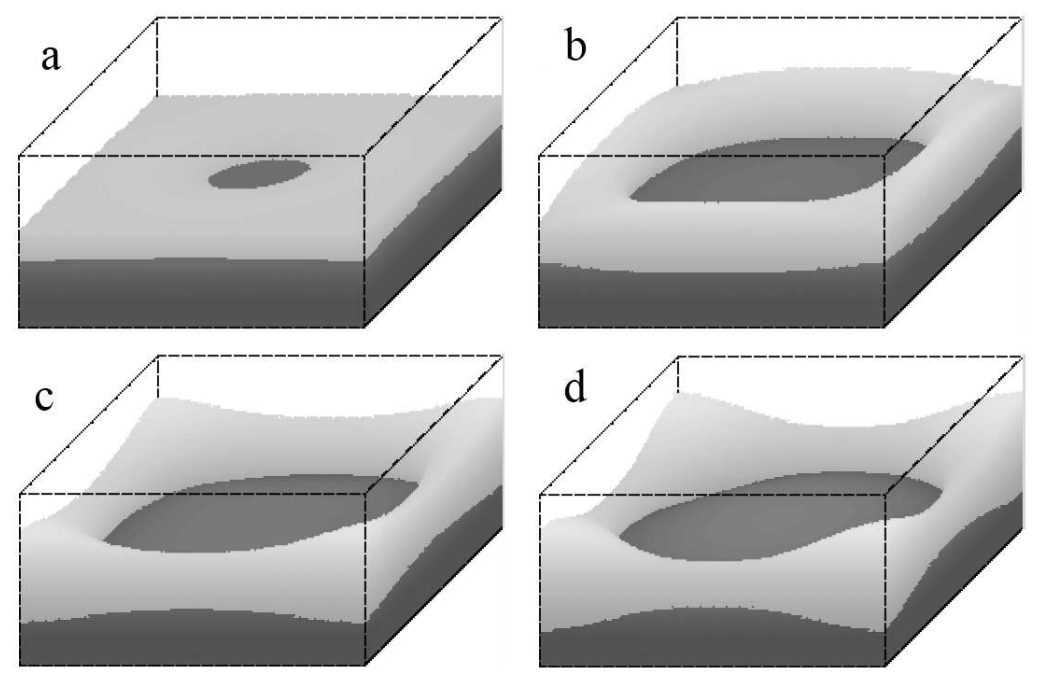

Figure 13: The rupture of the thin liquid film on the liquid substrate. $t=3450$ (a), 4450 (b), 4950 (c), 5550 (d). Lattice is $160 \times 160 \times 80$. 

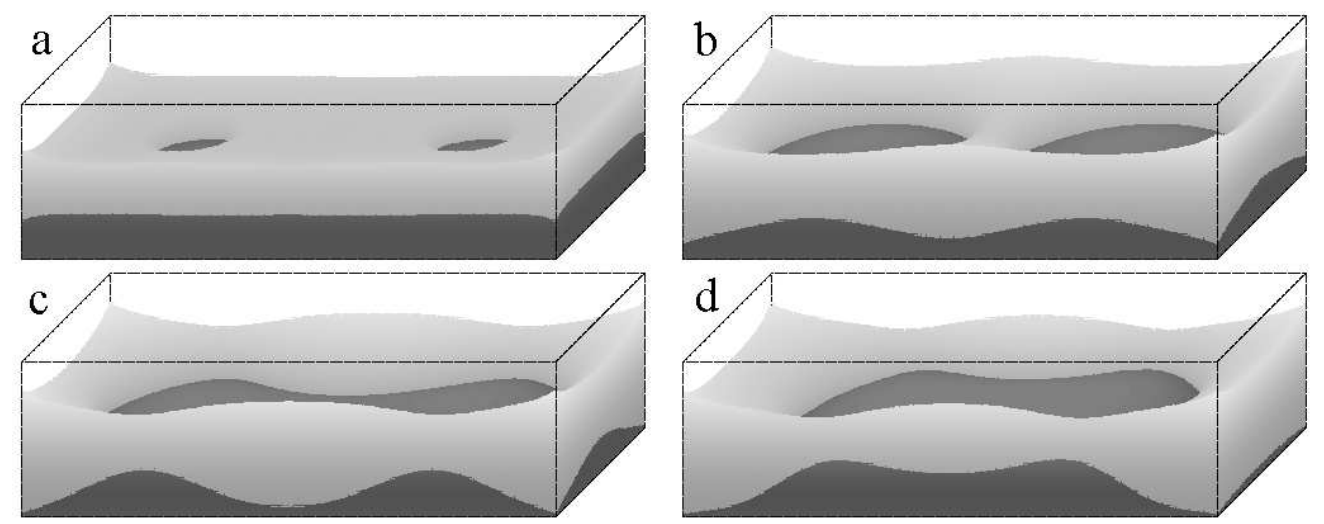

Figure 14: Merging of two holes in the film on the liquid substrate. $t=4300$ (a), 8000 (b), 13600 (c), 35200 (d). Lattice is $384 \times 160 \times 112$.

The results of simulation of the film on the liquid substrate in the case of two initial holes are shown in Fig. 14.

In the case of the no-slip and impermeability boundary conditions the thin layers are present at the surface of the walls permanently. The oscillations of the liquid flows attenuate in time. Of course, the shape of the liquid film shown in Fig. 14d corresponds to a transient state, which approaches the steady state asymptotically. It is observed that the flows of liquid films for single-layer on the nonwettable substrate and for two-layer arrangements are qualitatively similar.

\section{Conclusions}

The lattice Boltzmann equation method is used for three-dimensional computer simulations of the rupture of thin liquid films on the surface of solid and liquid substrates due to the thermocapillary effect (Marangoni effect) for prescribed temperature distributions.

This paper presents the results of numerical and physical experiments on the rupture of thin liquid films spread over a heavier immiscible liquid substrate in a rectangular cell. The drastic variations of the shape of the holes in the liquid film occur due to the action of surface tension and the interaction with the wettable walls of the rectangular cell.

The interaction of two holes has been studied experimentally as well as in computer simulations. Both holes increase with time, and the liquid bridge separating the two holes is destroyed after some time. After merging, the resulting single hole evolves toward the ultimate stationary oval-shaped state.

Evolution of holes in liquid films is simulated for two-layer systems of immiscible fluids. Gravity plays an important role in this process. A reasonable qualitative agreement is observed between experimental and numerical results. 


\section{Acknowledgments}

This work was supported in part by the Russian Foundation for Basic Research (grant No. 13-01-00526) and by the Ministry of Education of Russian Federation.

\section{References}

[1] A. Scheludko, Thin liquid films, Adv. Colloid Interface Sci. 1 (1967) 391-464.

[2] A. Scheludko, E. Manev, Critical thickness of rupture of chlorbenzene and aniline films, Trans. Faraday Soc. 64 (1968) 1123-1134.

[3] I.B. Ivanov, B. Radoev, E. Manev, A. Scheludko, The theory of the critical thickness of rupture of thin liquid films, Trans. Faraday Soc. 66 (1970) 1262-1273.

[4] R.V. Craster, O.K. Matar, Dynamics and stability of thin liquid films, Rev. Mod. Phys. 81 (3) (2009) 1131-1198.

[5] D. Bonn, J. Eggers, J. Indekeu, J. Meunier, E. Rolley, Wetting and spreading, Rev. Mod. Phys. 81 (2) (2009) 739-805.

[6] Yu.K. Bratukhin, A.L. Zuev, K.G. Kostarev, A.V. Shmyrov, Stability of a steady-state discontinuity of a fluid layer on the surface of an immiscible fluid, Fluid Dynamics 44 (3) (2009) 340-350.

[7] A.L. Kupershtokh, Three-dimensional simulations of two-phase liquid-vapor systems on GPU using the lattice Boltzmann method, Numerical Methods and Programming: Section 1. Numerical methods and applications 13 (2012) 130-138.

[8] Y.-H. Qian, S. Chen, Finite size effect in lattice-BGK models, International Journal of Modern Physics C 8 (4) (1997) 763-771.

[9] R. Zhang, H. Chen, Lattice Boltzmann method for simulations of liquid-vapor thermal flows, Phys. Rev. E. 67 (6) (2003) 066711.

[10] A.L. Kupershtokh, D.A. Medvedev, D.I. Karpov, On equations of state in a lattice Boltzmann method, Computers and Mathematics with Applications 58 (5) (2009) 965-974.

[11] A.L. Kupershtokh, Simulation of flows with liquid-vapor interfaces by the lattice Boltzmann method, Vestnik NGU (Quarterly Journal of Novosibirsk State Univ.), Series: Math., Mech. and Informatics 5 (3) (2005) 29-42.

[12] A.L. Kupershtokh, D.I. Karpov, D.A. Medvedev, C. Stamatelatos, V.P. Charalambakos, E.C. Pyrgioti, D.P. Agoris, Stochastic models of partial discharge activity in solid and liquid dielectrics, IET Science Measurement and Technology 1 (6) (2007) 303-311.

[13] Y.H. Qian, D. d'Humières, P. Lallemand, Lattice BGK models for Navier - Stokes equation, Europhys. Lett. 17 (6) (1992) 479-484.

[14] P.L. Bhatnagar, E.P. Gross, M.K. Krook, A model for collision process in gases. I. Small amplitude process in charged and neutral one-component system, Phys. Rev. 94 (3) (1954) 511-525.

[15] A.L. Kupershtokh, New method of incorporating a body force term into the lattice Boltzmann equation, Proc. of the 5th International EHD Workshop, Poitiers, France, 2004, pp. 241-246.

[16] A.L. Kupershtokh, Incorporating a body force term into the lattice Boltzmann equation, Vestnik NGU (Quarterly Journal of Novosibirsk State Univ.), Series: Math., Mech. and Informatics 4 (2) (2004) 75-96.

[17] A.L. Kupershtokh, Criterion of numerical instability of liquid state in LBE simulations, Computers and Mathematics with Applications 59 (7) (2010) 2236-2245. 
[18] J.M.V.A. Koelman, A simple lattice Boltzmann scheme for Navier-Stokes fluid flow, Europhys. Lett. 15 (6) (1991) 603-607.

[19] L. Chen, Q. Kang, Y. Mu, Y.-L. He, W.-Q. Tao, A critical review of the pseudopotential multiphase lattice Boltzmann model: Methods and applications, Int. J. Heat Mass Transfer 76 (2014) 210-236.

[20] A.L. Kupershtokh, A lattice Boltzmann equation method for real fluids with the equation of state known in tabular form only in regions of liquid and vapor phases, Computers and Mathematics with Applications 61 (12) (2011) 3537-3548.

[21] J.C. Bird, R. de Ruiter, L. Courbin, H.A. Stone, Daughter bubble cascades produced by folding of ruptured thin films, Nature 465 (2010) 759-762. 